\title{
Análise da qualidade de vida dos costureiros e sua relação com o vínculo empregatício
}

\author{
Quality of life analysis of dressmakers and its relation to employment
}

\author{
Mônica Negrão Gomes', Nick Dorneli de Carvalho', Renato Mitsunori Nisihara'
}

\begin{abstract}
RESUMO | Contexto: A cidade de Cianorte, localizada na região noroeste do Estado do Paraná, tem no vestuário sua atividade industrial mais representativa, abrigando uma população expressiva de costureiros, os quais exercem sua profissão em âmbito doméstico, em facções ou em empresas. As exigências do trabalho na indústria de confecção podem caracterizar um expediente altamente exaustivo, devido à atividade altamente repetitiva e monótona, ao estresse em relação à exigência de produtividade e à permanência de longos períodos na mesma posição durante a jornada de trabalho. Objetivo: Considerando a grande importância que atualmente se atribui à qualidade de vida $(\mathrm{QV})$, o objetivo desta pesquisa foi avaliar a $\mathrm{QV}$ dos costureiros de Cianorte (PR) e avaliar se o vínculo trabalhista influencia tal medida. Métodos: $\mathrm{O}$ estudo tem desenho observacional, transversal e analítico, avaliando a QV de 301 costureiros de Cianorte. Para isso, foram aplicados dois questionários, um sociodemográfico e a versão traduzida e validada do questionário SF-36, que avalia a QV. Resultados: Nos domínios capacidade funcional, aspectos físicos, aspectos emocionais e aspectos sociais esses instrumentos tiveram índices satisfatórios. Contudo, nos aspectos dor, estado geral de saúde, vitalidade e saúde mental foi encontrada uma redução significativa da QV. Discriminando os diferentes trabalhadores, aqueles que exercem suas atividades em âmbito doméstico apresentaram piores resultados. Conclusão: Os costureiros de Cianorte apresentaram comprometimento de sua QV. Quando comparada entre os diferentes vínculos trabalhistas, verificou-se que os costureiros que trabalham em empresa apresentaram os melhores resultados na QV e os domésticos tiveram os índices mais insatisfatórios.
\end{abstract}

Palavras-chave I satisfação no emprego; qualidade de vida; trabalhadores; ambiente de trabalho.

ABSTRACT I Context: The clothing industry is the most representative field in the city of Cianorte, in the Northeast region of the state of Paraná, housing an expressive population of dressmakers who work in their households, factions, or companies. The work demands in the clothing industry can characterize highly exhausting work hours owing to to the extremely repetitive and monotonous activity, to the stress related to the demanded productivity, and the long periods in the same position during the work hours. Objective: Considering the major importance attributed to quality of life (QOL) nowadays, the objective of this study was to assess the QOL of dressmakers in Cianorte (PR), and observe if the working relationship influences such measure. Methods: This is an observational, cross-sectional, and analytical study that assesses the QOL of 301 dressmakers in Cianorte. Two questionnaires were applied, being one sociodemographic survey and one translated and validated version of the SF-36 questionnaire, which analyzes QOL. Results: In the domains of functional capacity and on physical, emotional, and social aspects, these instruments presented satisfactory indexes. However, concerning pain, general health status, vitality, and mental health, a significant reduction in QOL was found. Distinguishing different workers, those working in the household presented with the worst results. Conclusion: The QOL of dressmakers in Cianorte was compromised. The comparison between different working relationships showed that dressmakers working at a company presented with the best QOL results, and those in the household had the most unsatisfactory ones.

Keywords I work satisfaction; quality of life; workers; working environment. 


\section{INTRODUÇÃO}

A cidade de Cianorte, localizada na região noroeste do Estado do Paraná, tem no vestuário sua atividade industrial mais representativa. É o principal município produtor de confecções têxteis da região e, hoje, é considerado um grande aglomerado produtor de moda do Sul do país, sendo reconhecido como a "Capital do Vestuário". A produção mensal é de mais de 5 milhões de peças ${ }^{1}$ de roupas, e cerca de $20 \%$ de todo o jeans comercializado no país. Seu polo industrial de confecções conta com mais de 450 empresas e 600 grifes, empregando mais de 15 mil pessoas e movimentando uma série de setores paralelos, como corte e costura, bordados, lavagem de tecidos e cursos de moda, gerando cerca de 30 mil empregos indiretos em toda a região ${ }^{2,3}$.

As exigências do trabalho na indústria de confecção podem caracterizar um expediente altamente exaustivo, devido aos fatores como atividade altamente repetitiva e monótona, estresse em relação à exigência de produtividade, aos ruídos, à temperatura inadequada e à permanência por períodos longos na mesma posição durante a jornada de trabalho ${ }^{4,5}$. Teixeira et al. ${ }^{6}$ relataram que $94,4 \%$ dos costureiros sentem dores quando estão na máquina de costura. Notadamente, o ambiente de trabalho influencia na vida do trabalhador. Portanto, um ambiente insalubre não proporcionará um local saudável e produtivo, deixando de contribuir para o desenvolvimento socioeconômico ${ }^{7}$.

Krost $^{8}$ salienta que no decreto que regulamenta a Previdência Social encontra-se a Relação de Atividades Preponderantes e seus respectivos graus de risco. Dessas atividades, 27 são relacionadas à indústria têxtil/ vestuário, 22 são consideradas nível 3 (risco grave), 4 estão enquadradas no nível 2 (risco médio) e apenas 1 é classificada como nível 1 (risco leve), evidenciando o alto potencial de adoecimento a que esses trabalhadores estão submetidos.

A avaliação da qualidade de vida $(\mathrm{QV})$ pode ser feita levando-se em conta o grau de satisfação com a vida, de forma subjetiva, e pode mudar com o decorrer do tempo, uma vez que é uma condição dinâmica que reflete os eventos do cotidiano. A estimativa da $\mathrm{QV}$ em um determinado grupo de indivíduos pode sugerir novas condutas ou influenciar as políticas e as práticas para um determinado grupo social ${ }^{1}$. A relação entre o trabalho e a $\mathrm{QV}$ pode ser classificada como extremamente complexa, visto que, ao mesmo tempo em que o trabalho pode ser fonte de descontentamento e piora do estado de saúde, ele também pode gerar satisfação e bem-estar ${ }^{10}$.

O presente estudo teve o objetivo de verificar a $\mathrm{QV}$ dos costureiros na cidade de Cianorte e avaliar se o vínculo trabalhista influencia tal medida.

\section{MÉTODOS}

O presente estudo tem desenho observacional, transversal e analítico e foi aprovado pelo Comitê de Ética em Pesquisa da Sociedade Evangélica Beneficente de Curitiba (PR) (Parecer no 997.029/2015). A coleta de dados foi realizada com os costureiros da cidade de Cianorte (PR) entre os meses de maio e julho de 2015. Todos os participantes incluídos no estudo assinaram um Termo de Consentimento Livre e Esclarecido (TCLE) e responderam a um questionário sociodemográfico.

Como critérios de inclusão foram adotados: trabalhar como costureiro na cidade de Cianorte, ter idade superior a 18 anos e concordar em responder ao questionário, assinando o TCLE. Os critérios de exclusão utilizados foram: não se enquadrar nos critérios de inclusão, ter alguma doença grave diagnosticada, ter doença psiquiátrica previamente diagnosticada ou estar afastado da profissão por mais de duas semanas no período da entrevista.

Não há dados sobre o número exato de costureiros de Cianorte. Porém, os valores encontrados variam de 5.835 a 8.146 (contabilizado pelo Sindicato dos Oficiais Alfaiates, Costureiras e Trabalhadores na Indústria de Confecção de Cianorte - SINDCOST, em 2014) ${ }^{11}$. Realizou-se o cálculo de amostragem mínima, por meio do cálculo amostral com poder de amostra superior a $90 \%$, obtendo-se amostra mínima estimada de 262 pessoas. O número total de costureiros entrevistados foi de 323 pessoas. Após a aplicação dos critérios de inclusão e exclusão, o número final de costureiros incluídos na pesquisa foi de 301 indivíduos.

De acordo com sua vinculação trabalhista, foram definidos como "trabalhadores em empresa" os costureiros que eram registrados de acordo com a lei em uma empresa consolidada como tal. Foram considerados como "trabalhadores em facções” aqueles que tinham suas funções em 
pequenas unidades produtivas, que apresentam formas de organização variadas, geralmente caracterizadas como uma atividade livre, precária e clandestina, contratados de forma terceirizada por outras empresas, muitas vezes de forma informal ${ }^{12}$. Costureiros que desenvolviam suas atividades em sua residência de forma autônoma foram considerados "trabalhadores domésticos".

Como principal instrumento, foi aplicado o questionário SF-36 (Medical Outcomes Study 36 - Item Short-Form Health Survey), que é um questionário genérico (de fácil administração e compreensão) de avaliação de QV. O SF-36 foi traduzido para português e validado, sendo composto por 11 questões e 36 itens que englobam 8 domínios, sendo eles: capacidade funcional (10 itens), limitações por aspectos físicos (4 itens), dor ( 2 itens), estado geral da saúde (5 itens), vitalidade (4 itens), limitações por aspectos sociais ( 2 itens), limitações por aspectos emocionais ( 3 itens), saúde mental (5 itens); e uma questão comparativa sobre a percepção atual da saúde e há um ano ${ }^{13}$.

Os dados foram coletados por conveniência nos estabelecimentos que tiveram maior acessibilidade, em locais de trabalho autônomo, facções ou empresas, por adesão voluntária dos costureiros e com consentimento dos empregadores. Para todo entrevistado foi explicado o objetivo da pesquisa e como deveriam ser preenchidos os questionários de forma autoaplicável e anônima. Em nenhum momento os participantes foram expostos a situações constrangedoras ou foram identificados na pesquisa.

Após a coleta, os dados foram planilhados com o auxílio do programa $\operatorname{Excel}^{\circledR}$ (Microsoft Office 2010) e submetidos à ponderação dos dados padronizada para esse questionário; em seguida, foi realizado o cálculo Raw Scale, que atribui uma nota quantitativa variando de 0 a 100 para cada domínio separadamente. Quanto maior a nota, melhor a QV do respondente. $\mathrm{Na}$ análise estatística foram aplicados os testes de Kolmogorov-Smirnov para verificação da normalidade dos dados; de Kruskal-Wallis para comparar as três categorias de trabalhadores dentro de um mesmo domínio; de Mann-Whitney para variáveis contínuas, para comparar as categorias dois a dois; e exato de Fisher ou do $\chi^{2}$ para variáveis categóricas, conforme apropriado. Todas as análises foram feitas utilizando-se o pacote estatístico Graph Pad Prism 5.0. Foram considerados com diferença significativa entre os grupos resultados obtidos com valor p menor ou igual a $5 \%$.

\section{RESULTADOS}

A Tabela 1 apresenta as características sociodemográficas (expressas em número absoluto e porcentagem) dos grupos estudados, como sexo, local onde trabalha, idade, se tem ou não plano de saúde, grau de escolaridade, tempo que exerce a profissão de costura, quantas horas trabalha por dia aproximadamente, se trabalha aos domingos e feriados, o período que predominantemente exerce a profissão e a renda mensal aproximada.

Verificou-se que as mulheres compõem a maioria da população pesquisada. Em relação à idade, a mediana encontrada foi de 40,5 anos (entre 18 e 71 anos), e a maioria não tem plano de saúde (80,3\%). Também se evidenciou que a maioria dos costureiros estudados trabalha em empresas $(60,4 \%)$, seguida pelos que trabalham em facções $(30,5 \%)$ e, por fim, os trabalhadores domésticos $(8,9 \%)$. Em relação ao tempo na profissão, a maior parcela dos costureiros $(89,3 \%)$ trabalha nessa atividade há mais de um ano. Dentre os que afirmam trabalhar aos domingos e feriados, $62,8 \%$ são domésticos, $20 \%$ são de empresas e $17,1 \%$ são de facções.

Dentre os domésticos que trabalham aos domingos e feriados, $86,3 \%$ também trabalham mais de 8 horas por dia e $22,7 \%$ trabalham nos períodos diurno e noturno. Em contrapartida, $18,1 \%$ deles afirmaram ganhar mais de 2 e até 4 salários mínimos, revelando uma porcentagem maior do que a média geral da população pesquisada, em que apenas 3,6\% responderam ganhar mais de 2 salários mínimos (Tabela 2).

Como apresentado na Tabela 2, os entrevistados apresentaram comprometimento nas categorias: estado geral de saúde, dor, vitalidade e saúde mental. Nos domínios capacidade funcional, aspectos físicos, aspectos emocionais e aspectos sociais, os costureiros tiveram índices satisfatórios de QV.

A Tabela 3 dispõe os resultados do QV SF-36, discriminado em locais de trabalho, em que fica evidenciado que, na maioria dos domínios, os trabalhadores domésticos têm uma pontuação significativamente menor que os outros dois grupos, o que indica uma pior QV nesses indivíduos. Quando avaliadas as diferenças para cada domínio entre os costureiros que trabalham em empresa, em facção e em sua residência, verificou-se alteração significativa para os domínios: vitalidade $(\mathrm{p}=0,004)$, aspectos emocionais $(\mathrm{p}=0,038)$, 
saúde mental ( $\mathrm{p}=0,0005)$, dor $(\mathrm{p}=0,0015)$ e aspectos sociais $(\mathrm{p}=0,04)$. Por outro lado, para os domínios capacidade funcional $(\mathrm{p}=0,69)$, aspectos físicos $(\mathrm{p}=0,11)$ e estado geral de saúde $(\mathrm{p}=0,06)$, não se verificou diferença significativa na QV por meio do teste de Kruskal-Wallis. Ao se comparar grupo a grupo (doméstico versus empresa, doméstico versus facção e empresa versus facção) para cada domínio, verificou-se que os costureiros de facção e de empresa divergem significativamente em vitalidade $(\mathrm{p}=0,0013)$ e em saúde mental $(\mathrm{p}=0,009)$, sendo que nos dois casos os trabalhadores em

Tabela 1. Dados sociodemográficos dos costureiros de Cianorte (PR).

\begin{tabular}{|c|c|c|c|}
\hline Questão & n (\%) & Questão & n (\%) \\
\hline Sexo & & \multicolumn{2}{|l|}{ Quanto tempo de profissão (meses) } \\
\hline Mulheres & $286(95)$ & Menos de 4 & $7(2,9)$ \\
\hline Homens & $15(4,9)$ & Entre 4 e 12 & $21(6,9)$ \\
\hline Onde trabalha & & Acima de 12 & $269(89,3)$ \\
\hline Empresa & $182(60,4)$ & Não responderam & $4(1,3)$ \\
\hline Facção & $92(30,5)$ & \multicolumn{2}{|l|}{ Trabalha quantas horas por dia } \\
\hline Domésticos & $27(8,9)$ & De 0 a 4 & $2(0,6)$ \\
\hline Idade (anos) & & Entre 4 e 8 & $87(28,9)$ \\
\hline $18-29$ & $50(16,6)$ & Acima de 8 & $196(65,1)$ \\
\hline $30-39$ & $77(25,5)$ & Não responderam & $16(5,3)$ \\
\hline $40-49$ & $107(35,5)$ & \multicolumn{2}{|l|}{ Trabalha aos domingos e feriados } \\
\hline $50-60$ & $38(12,6)$ & $\operatorname{Sim}$ & $35(11,6)$ \\
\hline Superior a 60 & $4(1,3)$ & Não & $264(87,7)$ \\
\hline Não responderam & $25(8,3)$ & Não responderam & $2(0,6)$ \\
\hline Tem plano de saúde & \multicolumn{3}{|c|}{ Período que predominantemente exerce a profissão } \\
\hline Sim & $52(17,2)$ & Somente pela manhã & $3(0,9)$ \\
\hline Não & $242(80,3)$ & Somente à tarde & $1(0,3)$ \\
\hline Não responderam & $7(2,3)$ & Somente noturno & $4(1,3)$ \\
\hline Grau de escolaridade & & Pela manhã e pela tarde & $282(93,6)$ \\
\hline Ensino fundamental incompleto & $63(20,9)$ & Pela manhã, pela tarde e à noite & $9(2,9)$ \\
\hline Ensino fundamental completo & $89(29,5)$ & Não responderam & $2(0,6)$ \\
\hline Ensino médio incompleto & $55(18,2)$ & \multicolumn{2}{|l|}{ Renda mensal aproximada* (salários mínimos) } \\
\hline Ensino médio completo & $77(25,5)$ & Entre 1 até 2 & $286(95)$ \\
\hline Ensino superior incompleto ou completo & $8(2,6)$ & Mais de 2 até 4 & $10(3,3)$ \\
\hline \multirow[t]{2}{*}{ Não responderam } & $9(2,9)$ & Mais de 4 & $1(0,3)$ \\
\hline & & Não responderam & $4(1,3)$ \\
\hline
\end{tabular}

*Salário mínimo considerando a vigência de 01 de janeiro de 2015 (Decreto no 8.381 - no valor de R\$ 788,00). 
facção foram os mais comprometidos. A comparação entre costureiro doméstico e de empresa foi a que apresentou maiores diferenças, sendo que nos domínios aspectos físicos $(\mathrm{p}=0,04)$, aspectos emocionais $(\mathrm{p}=0,011)$, saúde mental $(p=0,001)$, dor $(p=0,0004)$ e aspectos sociais $(p=0,021)$ os costureiros domésticos apresentaram QV significativamente pior (Tabela 3).

A comparação entre os trabalhadores domésticos e os de facção também revelou um maior comprometimento daqueles que exercem sua profissão em casa. Nos domínios saúde mental $(\mathrm{p}=0,041)$ e dor $(\mathrm{p}=0,0007)$, os costureiros domésticos obtiveram um escore significativamente inferior, comparados aos trabalhadores em facção. Na avaliação geral, não foi encontrada diferença significativa para as dimensões capacidade funcional e estado geral de saúde.

\section{DISCUSSÃO}

O estudo da QV de costureiros, comparando os diferentes grupos formados pela vinculação trabalhista, ao nosso conhecimento, é pioneiro no Brasil. A indústria têxtil e de vestuário é uma das ocupações mais disseminadas espacialmente no mundo e se constitui em uma importante fonte de geração de renda e emprego, especialmente em países em desenvolvimento ${ }^{14}$. Em Cianorte, $55,4 \%$ da população empregada na indústria faz parte desse setor, notabilizado nacionalmente pela intensa atividade e pelo número de trabalhadores envolvidos. Em âmbito nível estadual, esse setor compõe $11,7 \%$ da mão de obra industrial, perdendo em número absoluto de empregos industriais apenas para produtos alimentícios, bebida e álcool etílico ${ }^{11}$.

Neste estudo, a maioria da população pesquisada foi composta por mulheres (95\%), representando uma porcentagem acima daquela encontrada por outros autores, a qual foi de aproximadamente $80 \%^{15,16}$. Consequentemente, na população de costureiros, tal predominância implica em uma maior frequência de doenças que afetam mais esse gênero, como, por exemplo, distúrbios psiquiátricos sem associação com abuso de substâncias psicoativas ou álcool ${ }^{17}$ e doenças musculoesqueléticas ${ }^{18}$. Cabe ressaltar que, principalmente na faixa de renda estudada, as mulheres são responsáveis também pelos afazeres domiciliares, caracterizando um quadro de dupla jornada trabalhista. Tal situação, associada aos demais fatores de risco e ao pouco tempo para relaxarem ou realizarem atividade física ${ }^{18}$, pode contribuir para a baixa $\mathrm{QV}$ observada no presente estudo.

É conhecido que as mulheres tendem a buscar com mais frequência os serviços de saúde. Verificou-se, neste estudo, que mais de $2 / 3$ dos costureiros pesquisados não tem plano de saúde (80,3\%). Dada a alta frequência de queixas de saúde observada nessa população, seria recomendável que as empresas e facções dispusessem de convênios para seus trabalhadores, conferindo maior

Tabela 2. Pontuação obtida para cada domínio da qualidade de vida segundo questionário SF-36 e resultado do teste de Mann-Whitney.

\begin{tabular}{|c|c|c|c|c|c|c|}
\hline \multirow{2}{*}{ Domínios } & \multirow{2}{*}{ Mediana } & \multirow{2}{*}{ Menor valor } & \multirow{2}{*}{ Maior valor } & \multicolumn{3}{|c|}{ Valor $\mathrm{p}^{*}$} \\
\hline & & & & $E / F$ & $E / D$ & F/D \\
\hline Capacidade funcional & 90 & 5 & 100 & 0,86 & 0,7 & 0,8 \\
\hline Aspectos físicos & 100 & $\mathrm{O}$ & 100 & 0,33 & 0,04 & 0,19 \\
\hline Vitalidade & 70 & $\mathrm{O}$ & 100 & 0,0013 & 0,11 & 0,82 \\
\hline Saúde mental & 76 & 20 & 100 & 0,009 & 0,001 & 0,041 \\
\hline Dor & 67 & 10 & 100 & 0,83 & 0,0004 & 0,0007 \\
\hline Estado geral de saúde & 57 & 5 & 97 & 0,09 & 0,06 & 0,22 \\
\hline Aspectos sociais & 87,5 & 12,5 & 100 & 0,14 & 0,21 & 0,14 \\
\hline Aspectos emocionais & 100 & 0 & 100 & 0,31 & 0,011 & 0,09 \\
\hline
\end{tabular}

*Valor p referente ao resultado do teste de Mann-Whitney; E/F: empresa versus facção; E/D: empresa versus domésticos; F/D: facção versus domésticos. 
Tabela 3. Resultados do questionário SF-36 para cada domínio, discriminado de acordo com o vínculo empregatício, e resultado do teste de Kruskal-Wallis.

\begin{tabular}{lcc|c|c} 
Domínios $\left(\mathrm{p}^{*}\right)$ & Mediana & Menor valor & Maior valor \\
\multicolumn{2}{c}{ Capacidade funcional $(\mathrm{p}=0,69)$} & & \\
\hline Empresa & 87,5 & 5 & 100 \\
\hline Doméstico & 85 & 15 & 100 \\
\hline Facção & 90 & 20 & 100 \\
\hline
\end{tabular}

Limitações por aspectos físicos ( $p=0,11)$

\begin{tabular}{lccc}
\hline Empresa & 100 & 0 & 100 \\
\hline Doméstico & 75 & 0 & 100 \\
\hline Facção & 100 & 0 & 100 \\
\hline Vitalidade $(p=0,004)$ & & & \\
\hline Empresa & 70 & 20 & 100 \\
\hline Doméstico & 55 & 0 & 95 \\
\hline Facção & 62,5 & 0 & 100
\end{tabular}

Saúde mental $(p=0,0005)$

\begin{tabular}{llll}
\hline Empresa & 76 & 24 & 100 \\
\hline Doméstico & 56 & 20 & 100 \\
\hline Facção & 68 & 20 & 100
\end{tabular}

\begin{tabular}{cccc}
\hline Dor $(p=0,001)$ & & & \\
\hline Empresa & 72 & 10 & 100 \\
\hline Doméstico & 46 & 10 & 100 \\
\hline Facção & 72 & 20 & 100 \\
\hline
\end{tabular}

Estado geral de saúde $(p=0,06)$

\begin{tabular}{lccc}
\hline Empresa & 62 & 12 & 97 \\
\hline Doméstico & 52 & 5 & 97 \\
\hline Facção & 57 & 27 & 95
\end{tabular}

\begin{tabular}{lccc}
\hline Aspectos sociais $(p=0,04)$ & & \\
\hline Empresa & 87,5 & 12,5 & 100 \\
\hline Doméstico & 75 & 37,5 & 100 \\
\hline Facção & 75 & 37,5 & 100
\end{tabular}

Limitações por aspectos emocionais $(p=0,03)$

\begin{tabular}{llll}
\hline Empresa & 100 & 0 & 100 \\
\hline Doméstico & 66,6 & 0 & 100 \\
\hline Facção & 100 & 0 & 100 \\
\hline
\end{tabular}

*Valores p referentes ao teste de Kruskal-Wallis. facilidade de acesso a esses serviços ${ }^{19}$. Por outro lado, os gestores da Secretaria de Saúde do Município devem estar em alerta para a demanda específica dessa população, atentando para as principais queixas elencadas no presente estudo.

Negri et al. ${ }^{18}$, em um estudo com 1.007 trabalhadores diagnosticados com lesões por esforços repetitivos/distúrbios osteomusculares relacionados ao trabalho (LER/DORT), realizado na região de Piracicaba (SP), evidenciaram que os domésticos apresentaram maior prevalência de LER/DORT, e os costureiros constituíram a quinta categoria mais acometida por essa complicação. Esse achado corrobora as queixas apresentadas pelos costureiros do presente estudo, principalmente o fato de serem os domésticos os mais afetados. Em relação a LER/DORT, evidencia-se sua prevalência no tronco e nos membros superiores ${ }^{20}$. Adicionalmente, é válido destacar que as doenças mais prevalentes em pacientes em reabilitação profissional, segundo o Instituto Nacional de Seguridade Social (INSS), são majoritariamente musculoesqueléticas (26\%), seguidas das psiquiátricas $(18,17 \%)$ e, por último, do trauma $(15,21 \%)$. As duas primeiras são justamente aquelas que os costureiros estão mais expostos, aumentando o risco desses profissionais desenvolverem doenças ${ }^{21}$.

Quanto ao item dor, este estudo obteve uma mediana de 67 pontos, indicando alta prevalência desse sintoma nesses operários. Tal achado ratifica os encontrados por Pacheco et al. ${ }^{15}$, que relataram a presença de dor em 99,9\% dos costureiros em um estudo feito na cidade de Inhumas (GO). Em outro estudo, os autores observaram que os trabalhadores que estão há mais de seis meses nessa ocupação apresentaram cerca de três vezes mais chances de desenvolver sintomatologia dolorosa em mais de uma região corporal ${ }^{6}$.

Silva e Repolês ${ }^{22}$, analisando a dor em costureiras da cidade de Ervália (MG), verificaram que a região dorsal e a lombar tiveram maior incidência de sintomas. Apesar da queixa de dor, os costureiros de Cianorte, no geral, não apresentaram limitação por aspectos físicos. Porém, os trabalhadores domésticos apresentaram índices inferiores de pontuação no quesito dor, revelando um maior comprometimento dos aspectos físicos.

Comparando-se os costureiros que trabalham mais de 8 horas por dia $(77,7 \%)$ com os que trabalham menos, observou-se que aqueles reclamam mais de dor, 
provavelmente devido a maior exposição aos fatores desencadeantes ${ }^{15}$. Quanto a limitações por aspectos físicos, não se observou diferença entre quem trabalha mais ou menos de 8 horas diárias.

Entre as vantagens do trabalho doméstico está a possibilidade de controlar as horas de trabalho. Trabalhando sozinho, o costureiro pode decidir sua jornada de trabalho, como e com que velocidade deve trabalhar, sem o controle direto da hierarquia fabril. Entretanto, tal autonomia pode levar a uma jornada exaustiva, em que os costureiros domésticos trabalham mais horas e possivelmente com ritmo mais intenso, a fim de concluir no prazo o trabalho contratado. Apenas em períodos de menor demanda é possível subordinar o ritmo do trabalho ao ritmo da casa ${ }^{23}$.

Dentre os domésticos que trabalham por mais de 8 horas diárias, $81,4 \%$ costumeiramente trabalham aos domingos e feriados e $25,9 \%$ trabalham de manhã, de tarde e à noite. Beltrão e Pena ${ }^{24}$, em uma análise multivariada, identificaram como preditores de ocorrência de a síndrome metabólica, o sedentarismo e o exercício da profissão em período noturno. Além disso, é possível que a profissão interfira em outras atividades pessoais, em especial as relacionadas à família. Adicionalmente, parte da clientela não demonstra respeito aos períodos de descanso familiar (horário das refeições, noite e finais de semana) para serem atendidos, interferindo no funcionamento do lar, uma vez que os atendimentos são feitos em cômodos da casa ${ }^{25}$. Todos esses fatores contribuem para que a $\mathrm{QV}$ desses trabalhadores seja inferior, quando comparada aos costureiros com vínculo empregatício.

Um aspecto vantajoso do uso do questionário de $\mathrm{QV}$ SF-36 validado é que ele possibilita comparações com outras populações. Cattai et al. ${ }^{26}$ aplicaram esse instrumento a pacientes em hemodiálise, com idade média de 51,2 anos, que apresentaram escore médio de 67 pontos para estado geral de saúde e 67,5 pontos na categoria dor. Dentre os costureiros avaliados no presente estudo, mais da metade teve escores menores que 58 pontos no estado geral de saúde, abaixo do observado em pacientes em hemodiálise nesse domínio. Tais números preocupam, pois refletem uma $\mathrm{QV}$ abaixo do esperado para esse grupo de trabalhadores.
Um fator que pode contribuir para melhorar a $\mathrm{QV}$ para esses trabalhadores pode ser a aplicação da ginástica laboral, mais comum em empresas, possibilitando uma atenuação das consequências da ergonomia inadequada. Atribui-se alguns benefícios a essa prática, entre eles: redução da queixa de dor em 40 a 64\%, aumento da produtividade em 17 a $39 \%$, diminuição do número de acidentes no trabalho em 20 a 46\%, diminuição da ausência e dos afastamentos do trabalho em 70 a $86 \%{ }^{27}$.

Nesse contexto, recomenda-se que, uma vez realizado o diagnóstico dos possíveis riscos que essa profissão pode exercer sobre a $\mathrm{QV}$, faz-se necessária uma intervenção no ambiente físico, na organização e nos postos de trabalho. Entre as propostas, sugere-se uma melhora no desenho dos equipamentos, visando uma função ergométrica mais adequada, ginástica laboral, incentivo à prática de exercícios físicos e regulamentação dos trabalhadores informais.

Como limitação do presente estudo, elencamos o menor número de costureiros domésticos. Tal fato se deve à dificuldade de se conseguir contato com esses profissionais, uma vez que o acesso é mais difícil, visto que não existe um cadastro disponível desses trabalhadores.

\section{CONCLUSÃO}

Conclui-se que a QV dos costureiros da cidade de Cianorte, avaliados pelo questionário SF-36, apresentou comprometimento nos aspectos, dor, estado geral de saúde, vitalidade e saúde mental. Por outro lado, nos domínios capacidade funcional, aspectos físicos, aspectos emocionais e aspectos sociais eles tiveram índices satisfatórios. Quando comparada a QV entre costureiros de diferentes vínculos trabalhistas, verificou-se que os domésticos, em uma análise global, têm a QV mais prejudicada e os que trabalham em empresa apresentaram os melhores resultados na QV por meio do questionário SF-36. Dessa maneira, percebe-se como o trabalho pode influir a $\mathrm{QV}$ desses profissionais, principalmente os setores mais informais, nos quais se constatou um prejuízo ainda maior. Sugere-se ainda que não são apenas as condições de ergonomia próprias do trabalho que interferem na $\mathrm{QV}$, mas sim todo o contexto em que ele é realizado. 


\section{REFERÊNCIAS}

1. Instituto Paranaense de Desenvolvimento EconômicoeSocial (IPARDES). Arranjo produtivo local do vestuário de Cianorte: nota técnica. Curitiba: Secretaria de Estado do Planejamento e Coordenação Geral; 2006.

2. Guia de Cianorte. [Internet] Do ouro verde a Capital do Vestuário [acesso em 2016 maio 11]. Disponível em: <http://guiadecianorte. com/sobre-cianorte.html>

3. Cianorte. [Internet] Moda em Cianorte [acesso em 2016 maio 11]. Disponível em: <http://www.cianorte.pr.gov.br/a-cidade/moda/ moda-em-shopping/>

4. Prado RR. Avaliação da qualidade de vida na indústria do vestuário: o caso de costureiras portadoras de lombalgias Dissertação [Mestrado em Desenho Industrial]. Bauru (SP): Universidade Estadual Paulista; 2006.

5. Ambrosi D, Queiroz MFF. Compreendendo o trabalho da costureira: um enfoque para a postura sentada. Rev Bras Saúde Ocup. 2004;29(109):11-9.

6. Teixeira DP, Paiva MT, Nunes MCR. Identificação de fatores de risco para LER/DORT em uma indústria de confecção da cidade de Ubá/MG [Apresentação no IV Workshop de Análise Ergonômica do Trabalho e I Encontro Mineiro de Estudos em Ergonomia;2009 junho; Viçosa, Brasil].

7. Sousa-Uva A, Serranheira F. Trabalho e Saúde/(Doença): o desafio sistemático da prevenção dos riscos profissionais e o esquecimento reiterado da promoção da saúde. Rev Bras Med Trab. 2013;11(1):43-9.

8. Krost O. O trabalho em "facções" do ramo têxtil/vestuário em Blumenau/SC: um estudo de caso sobre saúde e adoecimento. Rev Curso Direito UNIFACS. 2015;185:78-7.

9. Seidl EMF, Zannon CMLDC. Qualidade de vida e saúde: aspectos conceituais e metodológicos. Cad Saúde Pública. 2004;20(2):580-8.

10. Moreira MMS. Trabalho, qualidade de vida e envelhecimento Dissertação [Mestrado em Ciências na Área da Saúde Pública]. Rio de Janeiro: Fundação Oswaldo Cruz; 2000.

11. Instituto Paranaensede Desenvolvimento Econômicoe Social(IPARDES). Caderno estatístico do município de Cianorte. Curitiba: IPARDES;2016.

12. Pimentel LCT. O trabalho e o processo de saúde-doença das costureiras por facção - região metropolitana de Goiânia/2010 Dissertação [Mestrado em Ciências Ambientais e Saúde). Goiânia: Pontifícia Universidade Católica de Goiás; 2010.

13. Ciconelli RM,FerrazMB,SantosW,MeinãoI,QuaresmaMR. Tradução para língua portuguesa e validação do questionário genérico de avaliação de qualidade de vida SF-36. Rev Bras Reumatol. 1999;39(3):145-50.

14. Lima AMDS. As faces da subcontratação do trabalho: um estudo com trabalhadoras e trabalhadores da confecção de roupas de Cianorte e região Tese [Doutorado em Ciências Sociais]. Campinas: Universidade Estadual de Campinas; 2009.
15. Pacheco LF, Formiga CKMR, Aires AKR, Melo LDG, Salgado PC. Aplicação da cinesioterapia laboral no combate das doenças osteomusculares relacionadas ao trabalho (DORT) em costureiros. Rev Movimenta. 2009;2(4):129-36.

16. Freitas JD, Francisco AC, Aquino DS. Diagnóstico da qualidade de vida e qualidade de vida no trabalho do APL de vestuário de Maringá/Cianorte - PR. [Apresentação no $2^{\circ}$ Seminário de Extensão e Inovação da UTFPR - 40 SEI; 2014; Cornélio Procópio, Brasil].

17. Rabasquinho C, Pereira H. Género e saúde mental: uma abordagem epidemiológica. Análise Psicológica. 2007;25(3):439-54.

18. Negri JR, Cerveny GCO, Montebelo MIL, Teodori RM. Perfil sociodemográfico e ocupacional de trabalhadores com LER/ DORT: estudo epidemiológico. Rev Baiana de Saúde Pública. 2014;38(3):555-70.

19. Travassos C, Martins M. Uma revisão sobre os conceitos de acesso e utilização de serviços de saúde. Cad Saúde Pública. 2004;20(Suppl 2):S190-8.

20. Gonçalves AC, Silva JKC, Benedito FHA, Tercariol SG. Prevalência das principais patologias consideradas doenças osteomusculares relacionadas ao trabalho no Centro de Referência em Saúde do Trabalhador de Araçatuba/SP. Fisioter Bras. 2013;14(1):33-7.

21. Vacaro JE, Pedroso FS. Reabilitação profissional e a aposentadoria especial nas doenças ocupacionais. Rev Bras Med Trab. 2013;11(2):60-5.

22. Silva FG, Repolês R. Análise da dor e qualidade de vida (QV) em costureiras de confecções de pequeno porte na cidade de Ervália, MG. Anais SIMPAC. 2014;6(1):65-70.

23. Abreu ARP. Trabalho e qualificação na indústria de confecção. Estud Fem. 1993;1(2):293-305.

24. Beltrão FLL, Pena PGL. Associação entre síndrome metabolica e saúde no trabalho. Rev Bras Med Trab. 2013;11(1):3-18.

25. Almeida AB, Hirata SF, Onesti LA, Recortes do trabalho doméstico de costureiras e suas interfaces com a esfera familiar, social e política. Terra e Cultura. 2003;19(37):82-93.

26. Cattai GBP, Rocha FA, Junior NN, Pimentel GGA. Qualidade de vida em pacientes com insuficiência renal crônica - SF-36. Ciênc Cuid Saúde. 2007;6(2):460-7.

27. Oliveira JRG. A importância da ginástica laboral na prevenção de doenças ocupacionais. Rev Ed Fís. 2007;139:40-9.

Endereço para correspondência: Renato Nisihara - Rua Padre Anchieta, 2770 - Bigorrilho - CEP: 80730-000 - Curitiba (PR), Brasil -

E-mail: renatonisihara@gmail.com 